\title{
Preparation and Evaluation of Metronidazole Benzoate Periodontal Patches
}

\author{
Farhana Tasneem, Marzia Alam and Md. Saiful Islam Pathan \\ Department of Pharmacy, State University of Bangladesh, Dhanmondi, Dhaka-1205, Bangladesh
}

Received: March 18; Accepted: April 07, 2015; Published (Web); July 21, 2015

\begin{abstract}
Periodontitis is a serious gum disease that damages the soft tissue and destroys the bone that supports the teeth. According to WHO, globally 15-20\% of middle-aged (35-44 years) adults, suffer from severe periodontal (gum) diseases. Metronidazole benzoate is an antibacterial agent prescribed against different diseases including, periodontitis and several other protozoal infestations. The aim of this experiment was to formulate intrapocket periodontal patches of metronidazole benzoate to provide site specific therapeutic activity with very small loading dose. Nine (F1 to F9) formulations of metronidazole benzoate were prepared by solvent casting method using ethyl cellulose and Eudragit RLPO as polymers, dibutyl phthalate as plasticizer and alcohol and chloroform as solvent system. Various physicochemical evaluations including FTIR, Trinocular Microscopic images, folding endurance, surface $\mathrm{pH}$ and content uniformity were determined. All the batches revealed content uniformity between $94.0 \%$ to $98.0 \%$. It was observed that the thickness and weight of the films were directly proportional to the total solid content of the film. F1 showed the lowest thickness and weight $(232.5 \mu \mathrm{m}$ and $35.45 \mathrm{mg}$ respectively) and F9 displayed the highest $(864.7 \mu \mathrm{m}$ and $85.23 \mathrm{mg}$ respectively). Folding endurance was directly proportional to the content of plasticizer. Formulation F3 was considered as the best formulation based on its transparent appearance, folding endurance (>200 times), surface $\mathrm{pH}(6-7)$ and $97.5 \%$ content uniformity.
\end{abstract}

Key words: metronidazole benzoate, periodontal patch, periodontitis.

\section{Introduction}

Dental plaque is a microbial bio-film. It is a diverse microbial community found on the tooth surface embedded in a matrix of polymers of bacterial and salivary origin. When plaque is not removed adequately, it causes an accumulation of calculus (tartar - it has a yellow color) at the base of the teeth, near the gums (Reddy, 2011). Plaque and tartar eventually irritate the gums (www.nidcr.nih.gov). Prolonged accumulation of plaque and tartar on teeth tends to become more harmful. The bacteria cause inflammation of the gums that is called "gingivitis". When gingivitis is not treated, it can advance to "periodontitis", which is a serious gum infection that damages the soft tissue and destroys the bone that supports the teeth (Reddy, 2011). A research conducted by the American Academy of Periodontology has shown that periodontal disease is associated with several other diseases. For a long time it was thought that bacteria was the factor that linked periodontal disease to other disease in the body; however, more recent research demonstrates that inflammation may be responsible for the association. Periodontitis may lead to diabetes, heart diseases, stroke, osteoporosis, respiratory diseases, cancer, etc. Researchers found that men with gum disease were $49 \%$ more likely to develop kidney cancer, 54\% more likely to develop pancreatic cancer, and 30\% more likely to develop blood cancers. Periodontitis is common but largely preventable. It is usually the result of poor oral hygiene. Periodontics is the dental specialty that relates specifically to the care and maintenance of these tissues. It provides the support necessary to maintain teeth in function ( $w w w$.mayoclinic. org).

Antimicrobials are chemotherapeutic agents that reduce the amount of bacteria present at a particular site, either by specifically targeting certain organisms or by non-specifically reducing all bacteria. Antibiotics are a form of antimicrobial agents produced by or obtained from microorganisms that have the capacity to kill other 
microorganisms or inhibit their growth. Many chemotherapeutic agents can be delivered locally that helps achieve greater concentrations with reduced drug doses and systemic side effects. Slow releasing devices have the advantage of releasing antibiotics gradually and their effect can be directed to specific target area (Brunton et al., 2011).

The aim of this experiment was to formulate intrapocket periodontal patches (Figure 1) of metronidazole benzoate to provide site specific therapeutic activity with very small loading dose. Metronidazole benzoate itself is not systemically available following oral administration. It is hydrolysed to metronidazole and bezoic acid before or during absorption through the gut wall. Thus, only metronidazole can be detected in the circulation. It is bactericidal to anaerobic bacteria and is believed to disrupt bacterial DNA synthesis. They are not very effective against Actinobacillus actinomycetemcomitans infections but quite effective against obligate anaerobes such as Porphyromonas gingivalis and Prevotella intermedia (British Pharmacopoeia 2013; Brunton et al., 2011).

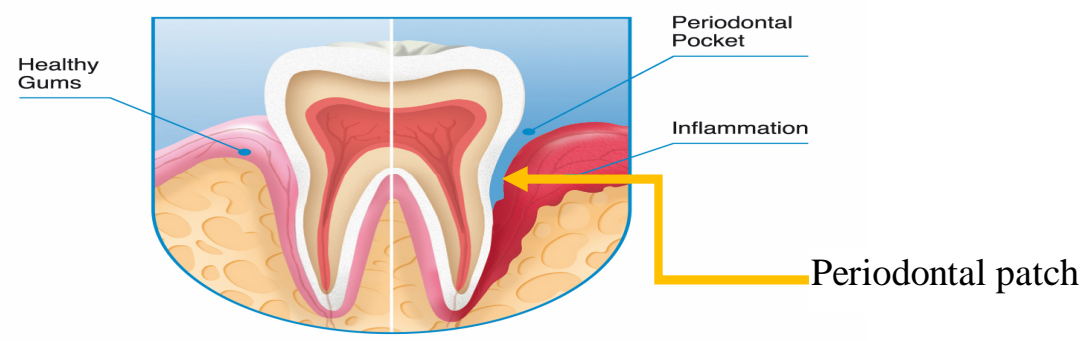

Figure 1. Periodontal pocket.

Clinically, metronidazole is used to treat necrotizing ulcerative gingivitis, adult periodontitis and rapidly progressive periodontitis. The recommended dose is 200 $\mathrm{mg}$ four times a day for 1 week or $400 \mathrm{mg}$ three times a day for 1 week (Brunton et al., 2011). This site specific patches with a very small loading dose may help achieve greater concentration of drug avoiding iatrogenic disease and thus better patient compliance.

\section{Materials and Methods}

Metronidazole benzoate was obtained as a gift from Opsonin Pharma. Ltd., Dhaka, Bangladesh. Ethyl cellulose (EC) (Ethocel standard 7 premiums) was obtained from Colorcon. Eudargit RLPO was obtained from Evonik and chloroform and ethanol were procured from Merck, India. Other ingredients used were of analytical grade.

Preparation of drug containing film: The solvent system was prepared by mixing ethyl alcohol and chloroform in a $25 \mathrm{ml}$ beaker followed by the addition of dibutyl pthalate (DBP). EC was added slowly with continuous stirring at $500 \mathrm{rpm}$ using magnetic stirrer. When EC was dissolved completely in the solvent, Eudragit RLPO was added slowly to the mixture. Lastly, accurately weighed metronidazole benzoate (BMET) was added. The mixture was stirred until a homogeneous mixture of drug and polymer was achieved. The homogeneous mixture was then poured into a petridish and dried in hot air oven at $55^{\circ} \mathrm{C}$ for 80 minutes. After the solvent evaporated, the films were cut into the desired size and shape and stored in air tight packets. Nine batches (F1 to F9) with different compositions were prepared (Table 1).

Physical evaluation of the films: Formulated films were subjected to the preliminary evaluations. Films with any imperfections, entrapped air or the films with differing thickness or weight were excluded from further studies.

Physical appearance: All films were visually inspected for colour, clarity, flexibility and smoothness.

Uniformity of thickness of the film: The thickness of each film was measured using micrometer (thickness tester) at different positions of the film and the average thickness was calculated.

Uniformity of weight of the films: Film (size of 10 $\mathrm{mm}^{2}$ ) was taken from different areas of the film. The weight variation of each film was determined. 
Folding endurance: The folding endurance of the films was determined by repeatedly folding a small strip of film of $20 \times 20 \mathrm{~mm}^{2}$ at the same place till it broke or folded up to 300 times, which is considered satisfactory to reveal good film properties (Alam et al., 2014).

Surface $p H$ : Periodontal films were left to swell for 1 hour on the surface of the agar plate, prepared by dissolving $2 \%(\mathrm{w} / \mathrm{v})$ agar in warm double distilled water under stirring and then pouring the solution into the petridish to gel/ solidify at room temperature. The surface $\mathrm{pH}$ was measured by means of $\mathrm{pH}$ paper placed on the surface of the swollen film. The mean of three readings was recorded (Alam et al., 2014).

Table 1. Formulation of metronidazole benzoate periodontal patches.

\begin{tabular}{lccccccccc}
\hline Ingredients & F1 & F2 & F3 & F4 & F5 & F6 & F7 & F8 & F9 \\
\hline Mertronidazole benzoate (mg) & 3.86 & 3.86 & 3.86 & 3.86 & 3.86 & 3.86 & 3.86 & 3.86 & 3.86 \\
Ethyl cellulose (mg) & 8.0 & 16.0 & 24.0 & 32.0 & 40.0 & 48.0 & 56.0 & 64.0 & 72.0 \\
Eudragit RLPO (mg) & 8.4 & 8.4 & 8.4 & 16.4 & 16.4 & 16.4 & 24.4 & 24.4 & 24.4 \\
DBP (ml) & 0.015 & 0.015 & 0.015 & 0.015 & 0.015 & 0.015 & 0.015 & 0.015 & 0.015 \\
Ethanol (ml) & 0.05 & 0.05 & 0.05 & 0.05 & 0.05 & 0.05 & 0.05 & 0.05 & 0.05 \\
Chloroform (ml) & 0.25 & 0.25 & 0.25 & 0.25 & 0.25 & 0.25 & 0.25 & 0.25 & 0.25 \\
\hline
\end{tabular}

Drug content uniformity: A film of known weight was taken in $10 \mathrm{ml}$ volumetric flask and dissolved in ethyl alcohol. $1 \mathrm{ml}$ of this solution was taken in $25 \mathrm{ml}$ volumetric flask and diluted with phosphate buffer ( $\mathrm{pH}$ 7.4).The solution was then filtered with Whatman filter paper no.1 and absorbance was recorded at $232 \mathrm{~nm}$. The polymer solution without drug served as blank.

\section{Results and Discussion}

\section{Evaluation of polymeric films}

Physical appearance: Polymers used for the fabrication of periodontal films showed good film forming property and reproducibility. The fabricated films were elastic and smooth. The films F1, F2, F3 were transparent; F4 and F5 were translucent and F6, F7, F8 and F9 were found to be opaque. This indicates that as the amount of polymers increases the films transparency decreases.

Weight variation: Drug loaded films $\left(1 \mathrm{~cm}^{2}\right)$ were tested for weight uniformity and the results are presented in tabulated structure (Figure 2). Lesser RSD value indicated that the films were uniform in weight. The weight of films are in the order F8> F9> F7> F6> F5> F4> F3> F2 $>$ F1. It was observed that F1 had the lowest weight and F8 had the highest weight. The formulated films had mass variation within the range of pharmacoepial specifications.
Thickness: The thickness of each film was measured at different points and average thickness with SD was calculated. The results are given below in figure 3. The data of the film thickness indicates that there was significant increase in thickness in the formulations. The order of thickness was F9>F8>F7> F6>F5>F4>F3> F2> F1. It has been observed that F1 and F9 showed the minimum and maximum thickness, respectively.

Folding endurance: The folding endurance value (Figure 4) was found to be in the following order: F7 < F6 $<\mathrm{F} 5<\mathrm{F} 4<\mathrm{F} 2<\mathrm{F} 3$. F8 and F9 were too brittle which is due to small quantity of plasticizer present in the formulations while F1 was too thin and too sticky to carry out the test which is due to higher amount of plasticizer. This indicates that as polymer concentration increases folding endurance decreases, in other words, folding endurance is directly proportional to the amount of plasticizer.

Surface $\mathrm{pH}$ : Surface $\mathrm{pH}$ of all the formulations was determined as described previously. All the formulations were found to have $\mathrm{pH}$ between 6 and 7. This reveals that the prepared films would not alter the $\mathrm{pH}$ of gingival fluid in periodontal pocket.

Drug content uniformity: Drug content uniformity ranged from 94 - 97.5\% which shows that there were no significant differences in drug content. But as compared to theoretical values, the estimated drug content was found to 
be less. This indicates that there was some amount of loss during processing.

Trinocular microscopic imaging of metronidazole benzoate periodontal patch: The surface of the film and distribution of polymer and drug within the film seemed to be uniform (Figure 5).

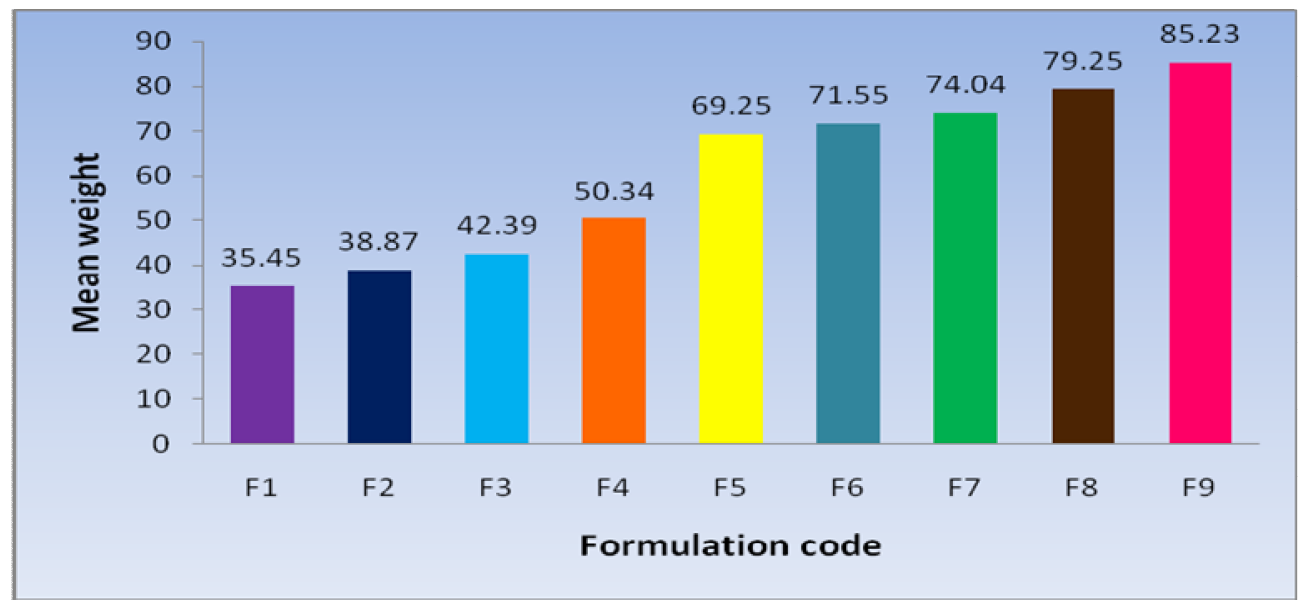

Figure 2. Comparative values of mean weight of films.

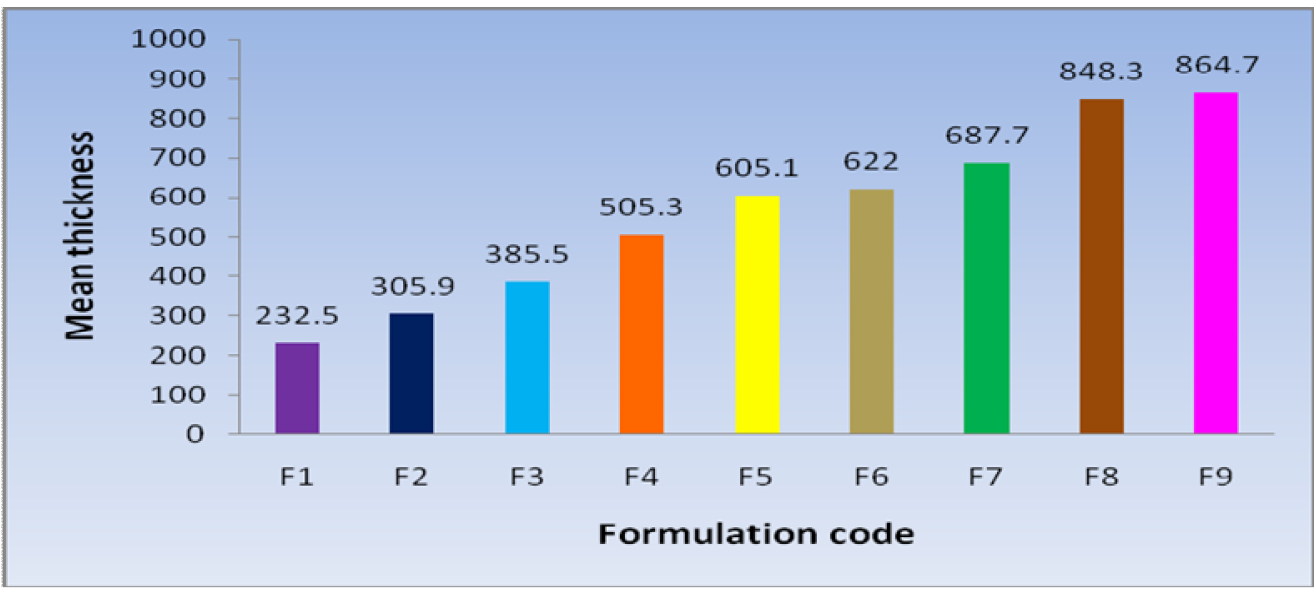

Figure 3. Comparative values of mean thickness of films.

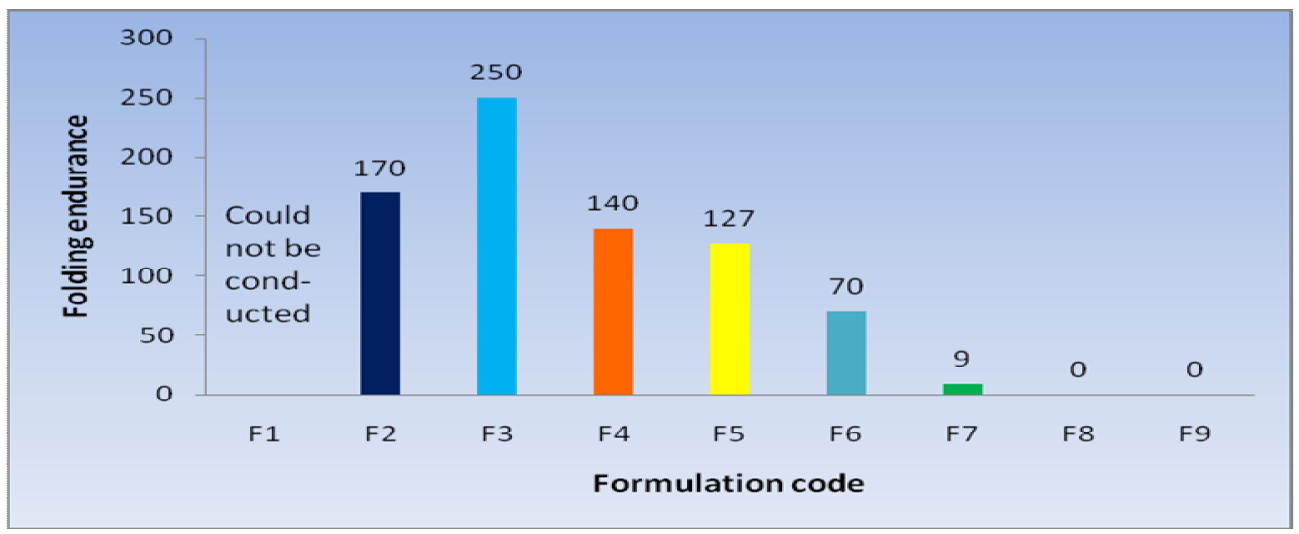

Figure 4. Comparative values of mean folding endurance of films. 
Table 2. Data of evaluation of polymeric films.

\begin{tabular}{|c|c|c|c|c|c|c|c|c|c|}
\hline \multirow[t]{2}{*}{ Evaluations } & \multicolumn{9}{|c|}{ Formulation code } \\
\hline & $\mathrm{F} 1$ & $\mathrm{~F} 2$ & $\mathrm{~F} 3$ & $\mathrm{~F} 4$ & F5 & F6 & F7 & F8 & F9 \\
\hline $\begin{array}{l}\text { Uniformity of } \\
\text { weights (mg) }\end{array}$ & $\begin{array}{c}35.45 \pm \\
0.974\end{array}$ & $\begin{array}{c}38.87 \pm \\
0.941\end{array}$ & $\begin{array}{c}42.39 \pm \\
0.952\end{array}$ & $\begin{array}{c}50.34 \pm \\
0.963\end{array}$ & $\begin{array}{c}69.25 \pm \\
0.972\end{array}$ & $\begin{array}{c}71.55 \pm \\
0.985\end{array}$ & $\begin{array}{c}74.04 \pm \\
1.317\end{array}$ & $\begin{array}{c}79.25 \pm \\
1.345\end{array}$ & $\begin{array}{c}85.23 \pm \\
1.519\end{array}$ \\
\hline Mean \pm RSD* & & & & & & & & & \\
\hline $\begin{array}{l}\text { Thickness }(\mu \mathrm{m}) \\
\text { Mean } \pm \mathrm{RSD}^{*}\end{array}$ & $\begin{array}{c}232.5 \pm \\
0.756\end{array}$ & $\begin{array}{c}305.9 \pm \\
0.865\end{array}$ & $\begin{array}{c}385.5 \pm \\
0.915\end{array}$ & $\begin{array}{c}505.3 \pm \\
0.926\end{array}$ & $\begin{array}{c}605.1 \pm \\
0.954\end{array}$ & $\begin{array}{l}622 \pm \\
0.992\end{array}$ & $\begin{array}{c}687.7 \pm \\
0.992\end{array}$ & $\begin{array}{c}848.3 \pm \\
1.187\end{array}$ & $\begin{array}{c}864.7 \pm \\
1.387\end{array}$ \\
\hline $\begin{array}{l}\text { Content uniformity } \\
(\%)\end{array}$ & 96.4 & 96.8 & 97.5 & 96.8 & 95.1 & 95.7 & 94.4 & 94.1 & 93.9 \\
\hline
\end{tabular}

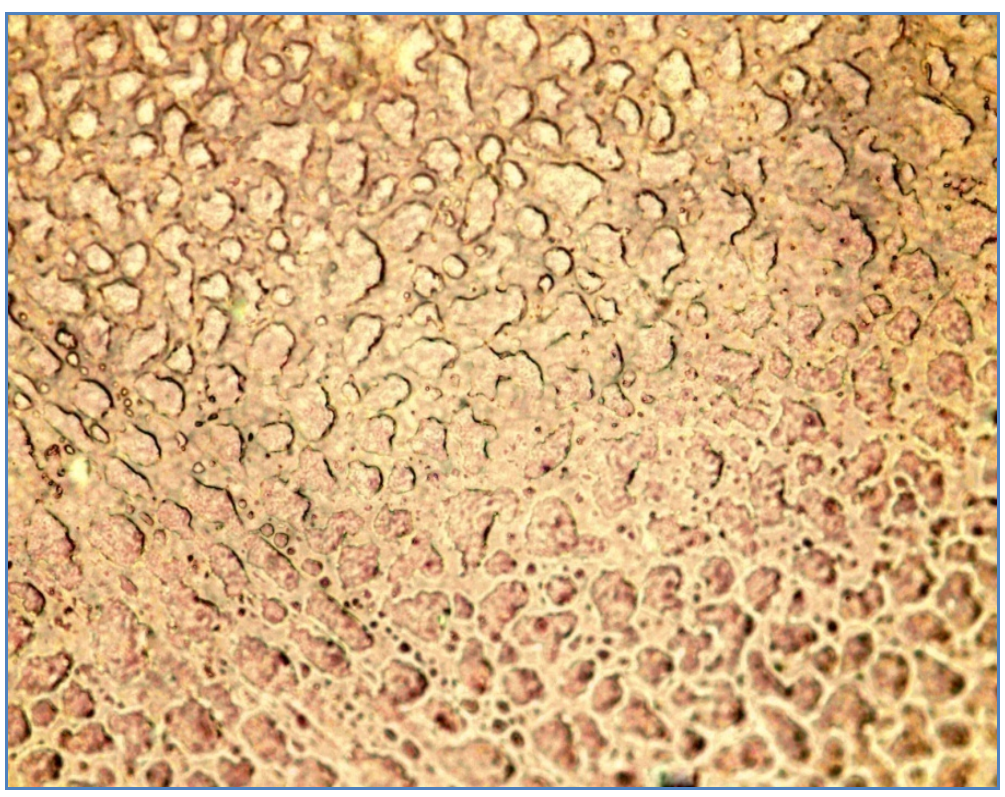

Figure 5. Trinocular microscopic imaging of metronidazole benzoate periodontal patch.

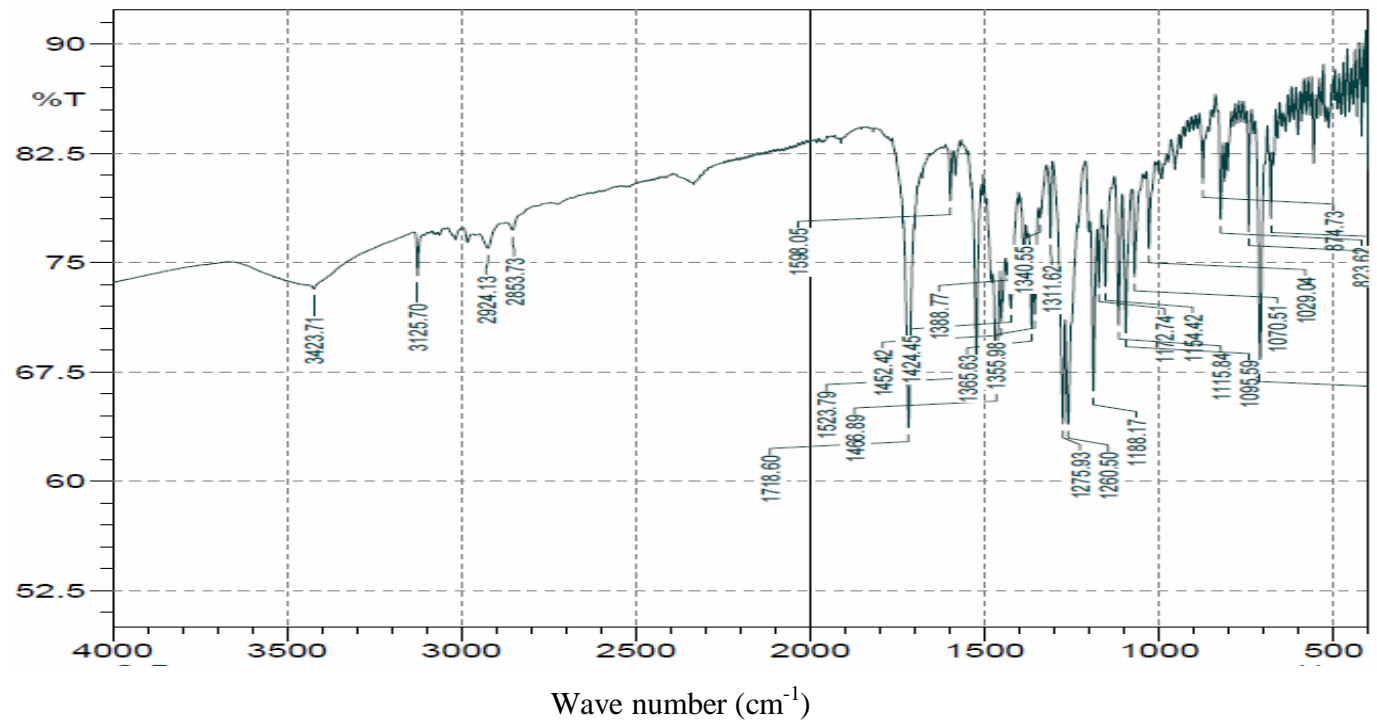

Figure 6. FTIR spectrum of pure metronidazole benzoate. 


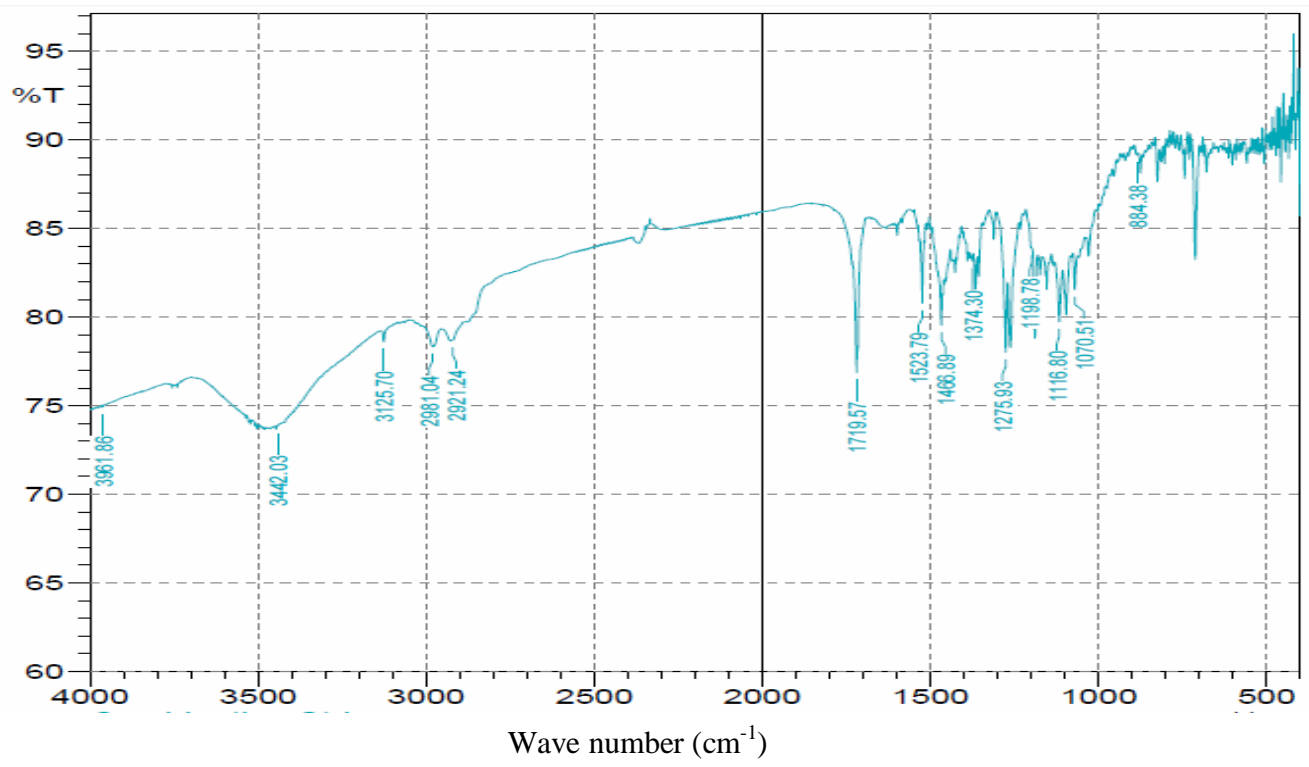

Figure 7. FTIR spectrum of metronidazole benzoate with polymers.

FTIR: It appears from the FTIR spectrum that there are slight changes in the spectrum of standard metronidazole benzoate and polymer combination (Figure 6 and 7). For pure metronidazole following peaks are seen: The major peaks shows slightly changed in the periodontal patch which needs further study.

\section{Conclusions}

In the present study, periodontal patches of metronidazole benzoate were prepared successfully using hydrophilic polymer - Eudragit RLPO, and Ethyl cellulose as hydrophobic polymer by simple solvent casting method. From the above study we can conclude that:

- Periodontal patches of Metronidazole benzoate can be prepared by solvent casting method.

- Hydrophobic polymer ethyl cellulose and hydrophilic polymer Eudragit RLPO can be used as combination polymers to make patches.

- Ethyl cellulose and Eudragit RLPO decreases the drug release with increase in its concentration.

Further study on formulation optimization, compatibility, stability, in-vitro release, release kinetic, antimicrobial property and scale-up studies is needed to confirm the appropriateness of these periodontal patches.

\section{References}

British Pharmacopoeia 2013. The British Pharmacopoeia Commission, London, UK.

Brunton, L.L., Chabner, B.A. and Knollman, B.C. 2011 Goodman \& Gilman's Pharmacological Basis of Therapeutics, $12^{\text {th }}$ edition.

Dumitrescu, A.L. 2010. Etiology and Pathogenesis of Periodontal Disease.

Marzia, A., Farhana, T. and M.S.I. Pathan. 2014. Formulation and evaluation of

swellable oral thin film of metoclopramide hydrochloride Bangladesh Pharm. J. 17, 102-112.

Newman, M.G., Takei, H., Klokkevold, R. and Carranza, F.A. 2015. Carranza's Clinical Periodontology $12^{\text {th }}$ edition.

Reddy, S. 2011. Essentials of Clinical Periodontology and Periodontics, 3rd edition.

http://en.wikipedia.org/wiki/Gingival_and_periodontal_pocket http://www.arestin.com/periodontal-gum-disease/stages.php http://www.dent-

wiki.com/foundations_of_periodontics/microscopicanatomy-of-alveolar-bone/

http://www.mayoclinic.org/diseasesconditions/periodontitis/basics/definition/con-20021679 http://www.medicalnewstoday.com/articles/241721.php http://www.nidcr.nih.gov/OralHealth/Topics/GumDiseases/Perio dontalGumDisease.htm

http://www.perio.org/consumer/other-systemic-diseases 